\title{
Calidad de los servicios financieros de las cooperativas rurales de la provincia de Tungurahua
}

DOI: https://doi.org/10.33262/ap.v3i4.1.121

\begin{abstract}
(c) (1) (3)
\end{abstract}
Quality of the financial services of the rural cooperatives of the province of Tungurahua: a view from the technological innovation

\author{
Walter Ramiro Jiménez-Silva. ${ }^{1}$, Iván Fernando Silva-Ordoñez. ${ }^{2}$ \& Mario Patricio \\ Padilla-Martínez. ${ }^{3}$
}

\begin{abstract}
Introduction. In a world of rapid change in which the financial services sector of rural cooperatives in the province of Tungurahua finds itself, the competitiveness of companies represents a crucial factor when it comes to facing each other, however, the absence of Technological innovation weakens the improvement of the quality of services in rural cooperatives. Objective. This research work has been prepared taking into account "technological innovation" in order to identify aspects that contribute to the improvement of the quality of financial services of rural cooperatives. Methodology. The data obtained, by means of the investigative instrument such as the survey statistically validated with Cronbach's Alpha with a result of 0.88 , with a questionnaire of 23 questions applied to 384 external clients, the level of correlation of the variables was estimated by the application of the Sperman test obtaining significant values at the 0.01 level. Results. It was determined that the degree of use of technological elements is relatively low.
\end{abstract}

\footnotetext{
${ }^{1}$ Universidad Técnica de Ambato, Facultad de Ciencias Administrativas, walterrjimenez@uta.edu.ec, ORCID https://orcid.org/0000-0001-8373-3549

${ }^{2}$ Universidad Técnica de Ambato, Facultad de Ciencias Administrativas, ivanfsilva@uta.edu.ec, ORCID https://orcid.org/0000-0002-2649-8578

${ }^{3}$ Universidad Técnica de Ambato, Facultad de Ciencias Administrativas, mp.padilla@uta.edu.ec, ORCID https://orcid.org/0000-0002-8325-2937
} 
Conclution. Which has a relative impact on the improvement of the quality of the services of rural cooperatives settled in the province of Tungurahua.

Keywords: Innovation, technology, quality, financial services, rural cooperatives

\section{Resumen}

Introducción. Dentro de un mundo de rápido cambio en el que se encuentra el sector de servicios financieros de las cooperativas rurales de la provincia de Tungurahua, la competitividad de las empresas representa un factor crucial a la hora de enfrentarse entre sí, sin embargo, la ausencia de innovación tecnológica debilita el mejoramiento de la calidad de los servicios en las cooperativas rurales. Objetivo. El presente trabajo de investigación ha sido elaborado tomado en cuenta "innovación tecnológica" con el propósito de identificar aspectos que contribuya para el mejoramiento de la calidad de servicios financieros de las cooperativas rurales. Metodología. Los datos obtenidos, por medio del instrumento investigativo como es la encuesta validada estadísticamente con al Alpha de Cronbach con un resultado de 0,88, con un cuestionario de 23 preguntas aplicadas a 384 clientes externos, se estimó el nivel de correlación de las variables mediante la aplicación del test de Sperman obteniendo valores significativos en el nivel 0,01. Resultados. Se pudo determinar que el grado de utilización de elementos tecnológicos es relativamente bajo. Conclusión. El cual impacta de forma relativa en el mejoramiento de la calidad de los servicios de las cooperativas rurales asentadas en la provincia de Tungurahua.

Palabras claves: Innovación, tecnología, calidad, servicios financieros, cooperativas rurales

\section{Introducción}

El presente artículo es una contribución para el desarrollo del proyecto de investigación titulado: "Modelo para mejoramiento de la calidad en las empresas de servicios de Tungurahua" adscrito a la Dirección de Investigación y Desarrollo (DIDE) Universidad Técnica de Ambato, con el código PFCAD12, un especial agradecimiento por su contribución a las tesista Gabriela Altamirano y Wendy Freire por su contribución con el trabajo de titulación "La innovación tecnológica y su impacto en el mejoramiento de la calidad de servicios en las empresas de servicios de Tungurahua".

La modernidad de los servicios aparece conjuntamente con la globalización tecnológica de la economía en los últimos 20 años, impulsada por las telecomunicaciones y servicios financieros; lo cual permite a las empresas diferenciarse de otras por la intangibilidad que los caracterizan, una interacción directa con el cliente optimizando el uso de tiempo y de recursos de ambas partes siendo una distinción de servicios de calidad (Tamarit, 2015). El progreso económico es impulsado por el desarrollo tecnológico, así como la evolución del mismo, esto incide en el comportamiento organizacional, producción, ciclo del producto, precio, optimización, tiempo, equipo de desarrollo interno y externo, el costo e 
inversiones (Núñez, 2013). En síntesis la evolución de una empresa está relacionado directamente a los avances tecnológicos que implementados y el crecimiento económico, así como su capacidad de solucionar problemáticas que la afecten directamente y a su entorno.

La innovación en los servicios se refieren a la implementación de nuevos productos o servicios en el mercado (a la implementación de nuevos métodos de interacción en el mercado), como una forma más amplia para contextualizar su alcance, lo que no siempre es aplicable, porque depende la competitividad de los productos, servicios o procesos de acuerdo a lo ya existente y de la oferta y demanda (Núñez \& Montalvo, 2015; Cardona et al., 2017). Así, la innovación tecnológica es impulsada por las necesidades divisadas con objetivos económicos alcanzables, adaptándose a los cambios del mercado por los nuevos comportamientos tecnológicos (Rodríguez et al., 2015); y es la base de la competitividad de las empresas pero representa un verdadero reto por su escasa inversión, al subestimar su potencial y buenas ideas que muchas de las veces son mal ejecutadas (Rodríguez, 2015). Por ello, es prioritario identificar los factores innovadores sistemáticos y coordinares en la tecnología son la bases para llegar a este fin.

Por otra parte, la calidad en los servicios en la gestión de la calidad es el eje clave para la mejora continua reflejándose en ciclo conocido como PDCA (Planear, Hacer, Verificar y Actuar), ejecutándolo en las cuatro etapas antes mencionadas; cabe considerar que el éxito de su eficacia está en el orden y desarrollo completo de dicho método (Gonzáles \& Vélez, 2015). Además, en toda organización o empresa las estrategias engloban el objetivo de potenciar la eficiencia y eficacia en los servicios ofertados mediante el análisis y auto evaluación de los mismos (Jiménez et al., 2008). Para Cuevas, 2016, considera como un factor enfático y determinante, para renovar la competitividad entre organizaciones, en cuanto a la adecuación de servicios, cubre necesidades de los consumidores y a la vez cumple con su satisfacción. En ese sentido, la calidad en los servicios se define como la satisfacción de necesidades y expectativas de los consumidores, se encamina a una referencia al ajuste de lo esperado por lo ejecutado.

Cabe agregar que, en toda organización la mejora continua en la calidad de sus servicios es fundamental, permite ser competitivo y es el principal factor que toma en cuenta el consumidor en su toma de decisión final (Jiménez et al., 2008). Por ello, gracias a la globalización de mercados y la alta competitividad, han transformado las necesidades de los consumidores en principales objetivos para las entidades financieras que compiten por un público objetivo; el cumplimento de este refleja a través de una gran ventaja competitiva a diferencia sus servicios con los de la competencia (Marino \& Moreno, 2014). Entonces, se debe conjugar aspectos tecnológicos en la calidad de servicios como una estrategia clave de grandes, medianas y pequeñas empresas apoyar a las empresas en su permanencia en el mercado, con el fin de captar eficientemente a los clientes por medio de servicios de calidad que satisfaga sus demandas

En esta dirección, los modelos de mejora de la calidad de servicios Servqual y Servperf son los más eficientes ya que permiten coordinar actividades y medir los resultados. La 
diferencia entre los dos es que se enfocan en la escala; Servqual discrepa entre las expectativas del servicio por recibir y las percepciones del servicio prestado; el modelo Servperf únicamente se guía a las percepciones de los consumidores (Arzola \& Mejías, 2007; Cardona et al., 2017). Entonces, se debe evaluar qué aspectos son pertinentes para aplicar en el modelo, sean las expectativas o percepciones de los clientes.

La problemática que se refleja es la ausencia de recursos tecnológicos para las cooperativas rurales de la provincia de Tungurahua, dificulta la existencia de innovación tecnológica en la planificación, limita las oportunidades de implementar mejoras en la de calidad de los servicios y mermando oportunidades.

La competitividad empresarial en la provincia de Tungurahua se ve afectado gravemente por la poca inversión extranjera, reduciendo las oportunidades de innovación tecnológica en la mejorar de la calidad de servicios, perdiendo presencia en este amplio mercado. Tanto el personal capacitado como sus servicios representan una alta inversión que impacta en los precios, lo que limita el acceso a las innovaciones tecnológicas en los servicio de las cooperativas rurales de las empresas. Por esta razón, el objetivo es plantear un modelo práctico de innovación tecnológica continuo para el mejoramiento de la calidad de servicios en las empresas de Tungurahua.

\section{Metodología}

La investigación fue dada a través de la correlación a través de la aplicación del test de Sperman, misma que estudia la relación que existente entre la variable dependiente (Calidad de servicios) y la independiente (Innovación Tecnológica); quiere decir se estudia la correlación entre dos variables, para de esa manera observar cómo se ve afectada cada una de estas y que es lo que más se destaca entre las entidades financieras como son los Bancos y Cooperativas de ahorro y crédito en existentes en la provincia de Tungurahua; el coeficiente de correlación recuperar información claves sin realizar ninguna manipulación de la misma como lo muestra en la tabla 1. Además, posee un enfoque cuantitativo, pues se basa en la recolección de datos a través de un instrumento, este se denomina encuesta. La misma, que tiene un cuestionario de 23 preguntas hechas a los clientes externos que pertenecen a las instituciones financieras de la provincia de Tungurahua, las cuales contestan a las dimensiones entre las variables por medio de una encuesta online.

Cabe agregar que, se validó el cuestionario a través del estadístico Alpha de Cronbach con un resultado de 0.877 que equivale a 0.88 . Ésta escala va de 0 a 1 , en donde los valores aceptados son desde 0.8 en adelante; razón por la cual este cuestionario se considera fiable al obtener un resultado de 0.88 . Y para este estudio se considera una muestra de 384 clientes de las entidades financieras (Bancos y Cooperativas de ahorro y crédito no rurales) de la provincia de Tungurahua, de una población de 29 entidades financieras entre Bancos y Cooperativas de ahorro y crédito (no rurales), de acuerdo al Instituto Nacional de Estadísticas y Censos ([INEC], 2012) de la CIIU (Clasificación Industrial Internacional Uniforme). 
Tabla 1

Correlación

\begin{tabular}{|c|c|c|c|c|c|c|c|}
\hline & Liderazgo & $\begin{array}{c}\text { Planificación } \\
\text { Estratégica }\end{array}$ & $\begin{array}{c}\text { Competencias } \\
\text { del recurso } \\
\text { humano }\end{array}$ & Procesos & Organización & $\begin{array}{c}\text { Satisfacción } \\
\text { del cliente }\end{array}$ & $\begin{array}{c}\text { Responsabilidad } \\
\text { social }\end{array}$ \\
\hline Tecnología actualizada &, $279^{* *}$ &, $223^{* *}$ &, $\mathbf{3 8 3}^{* *}$ &, $369^{* *}$ &, $349^{* *}$ &, $265^{* *}$ &, $298^{* *}$ \\
\hline $\begin{array}{l}\text { Aspectos físicos } \\
\text { calificados de forma } \\
\text { visual }\end{array}$ &, $198^{* *}$ &, $186^{* *}$ &, $201^{* *}$ &, $221^{* * *}$ &, $198^{* *}$ &, $202^{* *}$ &, $280^{* *}$ \\
\hline $\begin{array}{l}\text { Aspectos físicos en } \\
\text { relación con el servicio }\end{array}$ &, $198^{* *}$ & ,240** &, $262^{* *}$ &, $194^{* *}$ &, $245^{* *}$ &, $186^{* *}$ &, $190^{* *}$ \\
\hline $\begin{array}{l}\text { Adecuado horario de } \\
\text { atención }\end{array}$ &, $263^{* *}$ &, $205^{* *}$ &, $210^{* *}$ & ,242** & ,291** &, $216^{* *}$ &, $206^{* *}$ \\
\hline $\begin{array}{l}\text { Cuando se presenta un } \\
\text { problema ayuda a la } \\
\text { solución del mismo }\end{array}$ &, $208^{* *}$ &, $220^{* *}$ &, $189^{* *}$ &, $202^{* *}$ &, $297^{* *}$ &, $295^{* *}$ & ,240** \\
\hline $\begin{array}{l}\text { Servicio correcto y a la } \\
\text { primera vez }\end{array}$ &, $239^{* *}$ &, $248^{* *}$ &, $267^{* *}$ & ,286** &, $242^{* *}$ &, $262^{* *}$ &, $235^{* *}$ \\
\hline $\begin{array}{l}\text { Provee el servicio en el } \\
\text { tiempo ofrecido }\end{array}$ &, $218^{* *}$ &, $194^{* *}$ &, $264^{* *}$ &, $293^{* *}$ &, $259^{* *}$ & ,278** &, $241^{* *}$ \\
\hline $\begin{array}{l}\text { Brinda información } \\
\text { histórica }\end{array}$ & ,226** &, $144^{* *}$ &, $294^{* *}$ &, $211^{* *}$ & ,224** &, $202^{* *}$ &, $220^{* *}$ \\
\hline $\begin{array}{l}\text { El servicio que recibe el } \\
\text { cliente es eficiente }\end{array}$ &, $174^{* *}$ &, $142^{* *}$ &, $244^{* *}$ & ,246** &, $295^{* *}$ & ,250 $* *$ &, $207^{* *}$ \\
\hline $\begin{array}{l}\text { Disposición para } \\
\text { requerimientos del } \\
\text { cliente }\end{array}$ &, $169^{* *}$ &, $159^{* *}$ &, $118^{*}$ &, $132^{* *}$ &, $150^{* *}$ &, $199^{* *}$ &, $165^{* *}$ \\
\hline Confianza &, $109^{*}$ &, $290^{* *}$ &, $134^{* *}$ &, $167^{* *}$ &, $211^{* *}$ &, $119^{*}$ &, $283^{* *}$ \\
\hline $\begin{array}{l}\text { Los colaboradores } \\
\text { brindan atención de } \\
\text { forma individual }\end{array}$ &, 122 &, $187^{* *}$ &, $225^{* *}$ &, $200^{* *}$ &, $217^{* *}$ &, $222^{* *}$ &, $188^{* *}$ \\
\hline $\begin{array}{l}\text { Los colaboradores } \\
\text { poseen conocimiento } \\
\text { sobre mis necesidades }\end{array}$ &, $155^{* *}$ &, $256^{* *}$ &, $178^{* *}$ &, $226^{* *}$ &, $286^{* *}$ &, $309^{* *}$ &, $246^{* *}$ \\
\hline $\begin{array}{l}\text { Confianza sistemas de } \\
\text { pago }\end{array}$ &, $219^{* *}$ &, $188^{* *}$ &, $226^{* *}$ &, $225^{* *}$ &, $214^{* *}$ &, $304^{* *}$ &, $184^{* *}$ \\
\hline Servicio Posteriores &, $201^{* *}$ &, $233^{* *}$ &, $175^{* *}$ & ,288** &, $253^{* *}$ &, $212^{* *}$ &, $246^{* *}$ \\
\hline $\begin{array}{l}\text { Chatbots } \\
* * \text {. La correlación es sign }\end{array}$ & $\begin{array}{l}, 163^{* *} \\
\text { tiva en el ni }\end{array}$ & $\begin{array}{c}, 202^{* *} \\
10,01 \text { (bilateral) }\end{array}$ &, $122^{*}$ & ,230 ${ }^{* *}$ &, $261^{* *}$ & ,239* &, $199^{* *}$ \\
\hline
\end{tabular}

Fuente: Adaptado de Altamirano \& Freire (2019). La innovación tecnológica y su impacto en el mejoramiento de la calidad de servicios en las empresas de servicios de Tungurahua.

Las correlaciones que estadísticamente se muestran más representativas son:

La tecnología actualizada se relaciona con las competencias del recurso humano, procesos, organización y responsabilidad social; debido a que dentro de este sentido las diferentes actividades realizadas por las entidades financieras permiten una atención 
eficaz y eficiente para el cliente; proporciona así alta calidad y fácil accesibilidad hacia el servicio (Larrán et al., 2007).

La importancia de tener vigilada la tecnología es porque ayuda a realizar de forma sistemática, el análisis, la difusión y la explotación de las informaciones técnicas para la supervivencia y el crecimiento de la empresa, además alerta sobre cualquier innovación científica o técnica. Trata de analizar información desde diferentes frentes, como lo son el estudiar el comportamiento innovador de los competidores directos e indirectos y la tecnología incorporada a los mismos para aplicar nuevas tecnologías que permitan cerrar brechas tecnológicas para reaccionar oportunamente aprovechando oportunidades y contrarrestando amenazas (Corporación Tecnova UEE, 2011).

La actualización de la tecnología es muy importante para las organizaciones, por lo que es necesario realizar una vigilancia la cual ayudará a identificar si es preciso realizar cambios en las plataformas, además esto tendrá en cuenta identificar cualquier tipo de innovación que se esté dando en la tecnología. También, colabora a aprovechar las oportunidades que la tecnología ofrece para así poder ser competitivos frente a la competencia dando un servicio de calidad a los clientes.

Los aspectos físicos calificados de forma visual se relacionan con procesos, satisfacción del cliente y responsabilidad social; debido a que los clientes no únicamente se enfocan en el momento de recibir el servicio, también toman en cuenta y a la vez su satisfacción se cumple en la estructura física, la comodidad y el valor añadido que recibe por parte de la entidad financiera. Los aspectos físicos en relación con el servicio poseen una concordancia con planificación estratégica, competencia del recurso humano, organización, debido a que para la entrega del servicio los colaboradores y autoridades de la entidad financiera se siguen una visión por medio del cumplimiento de objetivos y metas.

La calidad el servicio es la conformidad del servicio prestado con las especificaciones y expectativas del cliente. Las organizaciones de servicio deben de determinar qué beneficios esperan recibir los clientes y de procurar producir los servicios que puedan satisfacer y si es posible exceder sus expectativas. Un servicio de calidad rara vez pasa inadvertido, pero en la prestación de servicios de calidad, lo más difícil es pasar del dicho al hecho (Lara, 2002a). La calidad del servicio es muy importante para las organizaciones ya que los clientes son el factor esencial para la calificación del servicio de cada empresa, además se debe tener en cuenta que si la empresa ofrece un buen servicio lograra fidelizar a sus clientes.

El adecuado horario de atención posee relación con liderazgo, procesos y organización; ya que para Eduarte (1999), dentro de lo que menos les gusta a los clientes son los horarios de atención; es por ello que las entidades deben crear estrategias que ayuden a mejorar procesos para una eficiente atención y que a partir de allí se pueda cumplir la satisfacción. 
Cuando se presenta un problema ayuda a la solución del mismo posee una mayor representatividad con organización, satisfacción con el cliente y responsabilidad social; ya que los problemas ya sean con soluciones previstas o nuevos problemas provenientes de consecuencias antes no consideras; deben ser observados de forma empírica con la realidad e identificados las deficiencias existentes; una vez llevado a cabo este proceso por parte de quienes conforman la entidad, se lleva a cabo la solución del mismo y pasa el principal objetivo de toda empresa como es la completa satisfacción del cliente (Acevedo \& Linares, 2009).

El servicio correcto y a la primera vez tiene una mayor relación con competencias del recurso humano, procesos y satisfacción del cliente; debido a que la calidad de servicios está directamente relacionada con que el servicio debe ser bueno y a la primera; ya que mientras mejor sea el servicio más alta es probabilidad de fidelización del cliente; es en este momento en donde nace la importancia de mejora y ajuste cada una de las necesidades de los clientes (Solórzano \& Aceves, 2013).

Proveer el servicio en el tiempo ofrecido tiene relación con competencias del recurso humano, procesos y satisfacción con el cliente; dado que en el caso de entidades financieras un factor importante es el tiempo; el éxito del mismo dependerá de los procesos dados por la entidad y la capacitación del personal en este caso se pondrá como ejemplo (cajeros); la eficiencia y efectividad tanto en el tiempo de espera como en el tiempo de interacción con el cliente (de Diago, 2013).

En todo el mundo, numerosas organizaciones se están dando cuenta que su éxito depende en gran medida del grado hasta el cual pueden satisfacer a sus clientes. Así el servicio al cliente aparece como el gran elemento diferenciador en el mercado, convirtiéndose en la estrategia en el producto, servicio y comercialización, imprescindible para sobrevivir en los mercados actuales (Botero \& Peña, 2006).

Las empresas en la actualidad fijan la importancia en la satisfacción del cliente ante todo de esto depende su éxito en el mercado y poder hacer frente a la competencia, además entregar un servicio de calidad se ha vuelto un reto para muchas empresas, porque ellos deben satisfacer las necesidades del cliente logrando tener mayores ingresos. Brindar información histórica posee relación con liderazgo, competencias del recurso humano y organización; debido a que la percepción que posee el cliente de la entidad financiera no únicamente es dada al momento del servicio, también toma como aspecto importante al servicio post - venta; por lo que actualmente las entidades financieras para cumplir con la disponibilidad de información histórica, pueden tomar en cuenta a un aspecto muy importante como es la Tecnología, ya que puede ser el medio por el cual el cliente podrá obtener la información mencionada en el momento y lugar en que lo requiera, a través de plataformas por ejemplo.

El servicio que recibe el cliente es eficiente posee mayor relación con procesos, organización y satisfacción del cliente; debido a que un manejo eficiente de cada uno de los procesos es el resultado de la reducción de costos y aumento en la calidad del servicio; 
en este caso el servicio financiero brindado hacia los clientes, para que pueda cumplirse lo esperado por lo cumplido. La disposición para requerimientos del cliente se relaciona con liderazgo, satisfacción con el cliente y responsabilidad social; por lo que los requerimientos se encuentran entre y después del proceso del servicio; es allí donde que se generan los momentos de verdad con el cliente ya que son los instantes de interacción y de poner en práctica estrategias trazadas anteriormente por los clientes.

La confianza posee relación con planificación estratégica, organización y responsabilidad social; debido a que la confianza parte desde las personas quienes dirigen las entidades financieras u organizaciones en sí, para que consecutivamente puedan impartir hacia sus colaboradores; mismos que aplicarán estrategias tanto dentro de la empresa como fuera de la misma para que la eficiencia se incremente a través de la confianza (Lockward, 2011). La calidad del servicio depende en gran medida del desempeño de los empleados porque es una interacción entre el cliente y el personal de la organización, cuando se mide la calidad del servicio lo que se suele medirse es la satisfacción hacia la calidad del servicio (Carlos et al., 2010).

Las empresas deben tener capacitados a su personal para que puedan dar un buen servicio, por lo mismo en las organizaciones se tiene mayor interacción con los clientes por lo cual el personal que esté a cargo de atención al cliente debe estar correctamente capacitados para que logren cumplir con las expectativas y satisfacción de los consumidores. Los colaboradores brindan atención de forma individual posee relación con competencias del recurso humano, organización y satisfacción del cliente; debido a que a mayor competencia del recurso humano mejor serán los tiempos de atención y respuesta a cada uno de los requerimientos, por lo que se pasa a un cumplimiento de expectativas y a una completa satisfacción de los mismos.

Los colaboradores poseen conocimiento sobre mis necesidades tiene relación con planificación estratégica, organización y satisfacción del cliente; por lo que previamente para cualquier resultado de satisfacción como punto principal se debe realizar un estudio sobre las necesidades de información tanto reales, expresadas y reconocidas; con el objetivo de precisar las principales variables de búsqueda, construir las estrategias más adecuadas por medio de una planificación estratégica y consecutivamente obtener necesidades de información actuales y potenciales (Salazar, 2011).

Confianza en los sistemas de pago posee relación con competencias del recurso humano, procesos, y satisfacción del cliente; debido a que dentro de las entidades bancarias la confianza es una creencia tan esencial que el rompimiento de la misma puede significar el quebranto de la misma actividad bancaria; por lo que las entidades de la mencionada actividad; deben aplicar tanto a través de canales tradicionales como virtuales de pago mecanismos estratégicos de seguridad para que los clientes puedan sentirse seguros y se pueda generar confianza en los mismos (Ampuño et al., 2015).

Servicios posteriores tiene relación con procesos, organización, y responsabilidad social; ya que el servicio postventa prepara ventas y hasta casi las aseguran, y respecto a ventas 
ya realizadas las fortalecen por medio de estrategias dadas por la entidad financiera; como aspecto muy importante tomado en cuenta para los actuales clientes son los valores éticos con un interés propio y común con el fin de establecer relaciones a largo plazo que aseguren la presencia de la entidad en el tiempo; como un ejemplo puede ser el servicio hacia la sociedad (Ferrer \& Martínez, 2006). Chatbots posee mayor relación con procesos, organización y satisfacción del cliente; ya que por medio de la inteligencia artificial que posee la misma puede brindar una conversación coherente en el momento que requiera el cliente ( 24 horas) y en determinados casos pueda realizar varios procesos (abrir una cuenta de ahorro); conseguir así un servicio personalizado y como resultado la total satisfacción del cliente (Fernández, 2019).

\section{Resultados}

Tanto el modelo SERVQUAL como el modelo SERVPERF hacen uso de cinco categorías, para evaluar la calidad de servicios, que son explicadas por (Marino \& Moreno, 2014; Núñez \& Montalvo, 2015): Confiabilidad, fiabilidad, seguridad, empatía, elementos tangibles. El modelo SERVPERF aplica veinte y dos variables que tienen que ver con lo que principalmente se enfoca el modelo como son las percepciones del cliente. Dicho modelo parte con la estructura de las categorías mencionadas anteriormente (confiabilidad, fiabilidad, seguridad, empatía y elementos tangibles) (Pérez, 2018), sin perder el enfoque de las percepciones del cliente dado por el modelo SERVPERF. Sin embargo, se debe considerar aspectos tecnológicos, administrativos y de marketing.

De acuerdo a Gil \& Luis ( 2011), mencionan que la innovación en las empresas es un aspecto fundamental para la calidad de servicios, por ello apoyan en la fidelización de sus clientes más valiosos. Por otro lado los autores Arzola \& Mejías (2007), establecen un modelo para la innovación en los servicios: liderazgo, planificación estratégica, procesos, organización, satisfacción de clientes, competencias del recurso humano y responsabilidad social. Sin embargo, no se toma en cuenta aspectos tecnológicos para su seguimiento. Y de acuerdo a (Zahera, 2003) detallan el siguiente modelo de gestión de la innovación tecnológica: desarrollo de productos, tecnología, innovación de procesos, estrategia tecnológica; incorporándose en cada uno: gestión de proyectos, organización, calidad/indicadores, recursos humanos, vigilancia y colaboración. Pero no se trata aspectos de marketing, y seguimiento a los clientes después de la adquisición de servicios, vital para un servicio de calidad.

Tras un análisis teórico, se plantea el siguiente modelo que permita la innovación tecnológica permanente para el mejoramiento de la calidad de servicios en las cooperativas rurales de Tungurahua. 
Figura 1

Modelo de innovación tecnológica permanente para el mejoramiento de la calidad de servicios

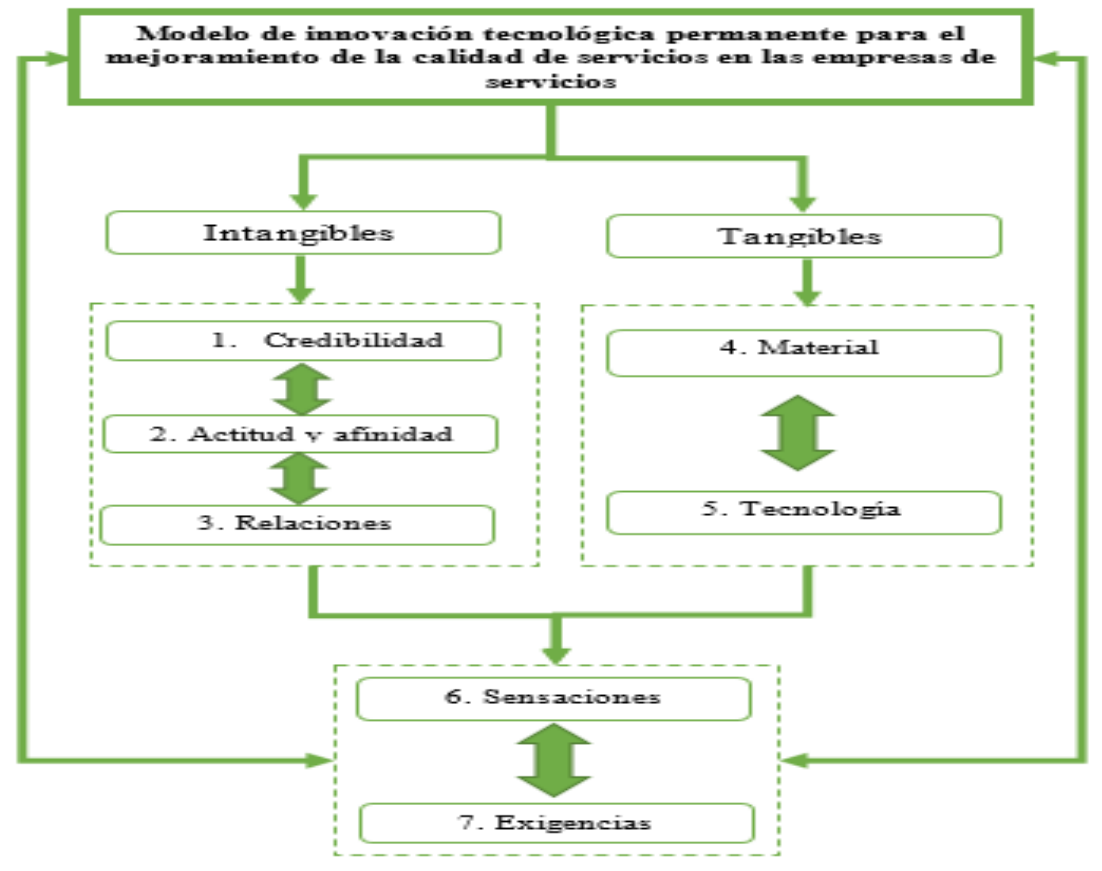

Fuente: Altamirano \& Freire (2019). La innovación tecnológica y su impacto en el mejoramiento de la calidad de servicios en las empresas de servicios de Tungurahua.

\section{Credibilidad}

Esta la dimensión de partida para el modelo, se establece los propósitos, objetivo y metas enfocadas s definición de los servicios teniendo presente la calidad física, corporativa e interactiva; toso esto se complementa con un cuidado análisis al público objetivo, dando como resultado la diferenciación por valoración competitiva en la calidad en los servicios que los clientes reciben.

La credibilidad ha recibido una especial atención en la literatura de marketing, debido a la notable influencia que ejerce en la consecución de relaciones duraderas y rentables. Es considerado como un conjunto de creencias colocadas en la mente del individuo, y derivadas de las percepciones que caracterizan a la marca, oferta, personal de ventas o al propio establecimiento donde se comercializan los productos o servicios, además la credibilidad es el grado de confianza que inspiran, por un lado, los empleados de contacto y, por otro, la imagen de la organización (Gutiérrez et al., 2010). Propósitos, objetivos y metas organizacionales. - el análisis de estos tres elementos definen la razón de ser, las metas orientadas a la visión empresarial, enfocados a brindar una atención de calidad complementándose con el seguimiento posterior; cuáles son las metas organizacionales y los resultados esperados en un marco temporal especifico. 
Definición de los servicios. - actividades y beneficios intangibles definidos así como ofertados en el mercado, mediante la interacción cliente, personal e instalaciones físicas de servicio; las cuales satisfagan los deseos, expectativas y/o necesidades. De igual forma los servicios engloban varias dimensiones de calidad de excelencia definidas por sus características propias beneficiosas, estas son: la calidad física de los servicios, la calidad corporativa que incide en la imagen de la empresa y la calidad interactiva, la cual es la interacción entre el personal y el cliente, y entre clientes.

Público objetivo. - la edad de los clientes, nacionalidad, ingresos, nivel socioeconómico, beneficios, modalidad de adquisición, estilo y personalidad son las variables a considerar para definir qué productos o servicios se deben ofertar y que estrategias a implementar. Creación de valor. - se define y especifica el plus diferenciador que genera las experiencias positivas con el cliente a través de los productos o servicios ofertados.

Momentos de contacto y momentos de verdad. - son las interacciones directas con el cliente, presencial o de forma online. En este punto se presente los momentos críticos que son las necesidades no satisfechas del cliente; no son aleatorios así como medibles, se deberá realizar un seguimiento, por ejemplo: solicitud de asesoramiento o información de forma presencia o digital por parte del cliente, el punto es el tiempo de espera y al cancelar el valor a pagar. Servicios y atención al cliente. - actividades encaminadas a que el cliente reciba el servicio en el momento adecuado y en el lugar apropia. Propiciando la fidelización del cliente, El punto de partida es la contante capacitación del personal en aspectos de amabilidad, responsabilidad, honestidad, escucha activa, respuesta rápida y solución de problemas.

\section{Actitud Y Afinidad}

Definir la cultura organizacional de la empresa que está constituida de los siguientes principios y valores organizacionales: respeto, trabajo en equipo, servicio al cliente, sentido de pertenencia y excelencia, los mismas que están alineadas con las estrategias de posicionamiento comercial que optimizan la precepción de la marca orientando a los clientes a la calidad en los servicios ofertados.

Cultura organizacional. - con bases a una actitud de servicio al cliente se fundamentan los principios y valores organizacionales, adaptables a necesidades del clientes del mercado actual. Entre ellos, son: los valores, el sentido de pertenencia, respeto al individuo, el trabajo en equipo, el servicio al cliente y la excelencia en mi trabajo.

La importancia de tener una actitud positiva hacia el cliente para lograr fidelizar al cliente y cumplir con sus expectativas. "La actitud es una predisposición aprendida a responder positiva o negativamente a cierto objeto, situación, institución o persona" (Tumino \& Komiejczuk, 2012). Posicionamiento: estrategias comerciales. - se enfoca en que los clientes reconozcan los beneficios, valores y atributos de los servicios que se oferten en el mercado, de esta forma se diseñan estrategias comerciales que cubran mercados fuera de la zona de confort de la empresa, posicionándose en la mente del cliente. Percepción de la marca. - enfocar acciones específicas para que el cliente reciba un servicio de 
calidad, que le transmitas sensaciones y una percepción positiva hecha por sus sentidos hacia la cooperativa logrando así su fidelización.

\section{Relaciones}

Las relaciones entre vendedores-clientes tales como desarrollo de directrices claves para el desempeño de la relación y su misma longevidad, factores de la relación a través del ciclo de vida, la calidad del servicio, atributos importantes de la relación, terminación de la relación, perspectiva del comportamiento del comprador, importancia de la iniciativa para efectos de la satisfacción del cliente (Gómez, 2010). Se establecen los parámetros de publicidad, comunicación integral y organizacional; esto permite diseñar estrategias que participan a detalle la calidad del servicio, estas se relacionan con los costes, beneficios, satisfacción de los clientes, la fidelidad de los consumidores entre otros; esto garantizara una larga relación con los clientes.

Publicidad. - herramienta fundamental del marketing, transmite un determinado mensaje positivo al cliente potencial sobre la oferta de sus productos, servicios, ideas u otros; para este fin se opta por estrategias creativas como la publicidad en medios publicitarios tradicionales (televisión, radio, prensa) o alternativos (eventos, anuncios en puntos de venta, redes sociales). Comunicación integral. - conjunto de herramientas estratégicas tanto internas como externas que posee todas las empresas, con el fin de posicionar así como controlar los mensajes al cliente y prevé potenciales escenarios futuros.

Percepción y calidad del servicio. - son las expectativas y experiencias que espera el clienta lo largo del servicio por parte de la institución; la retroalimentación permite medir el grado de satisfacción y el cumplimiento de lo ofertado mediante los productos y servicios; lo que conlleva ser un referente por parte de los clientes actuales hacia los potenciales.; las cooperativas rurales que implementa un trato cortes y amable, un léxico complementado con leguaje en el tono de voz apropiado a lo largo de su atención satisface las experiencia tanto del cliente como del personal.

\section{Material}

Se analizan aspectos de infraestructura, gestión de recursos, comunicación, personal y facilidades de pago. Estos elementos tangibles como las instalaciones físicas, el equipo personal y los materiales de comunicación son factores determinantes de la compra del servicio que se oferta.

Infraestructura. - son todas las instalaciones físicas de la empresa, su percepción pues refleja el orden y organización de la empresa, las que más trasmite una percepción positiva son: el parqueadero, amplitud de los pasillos, orientación, iluminación, distribución de los departamentos y señalización. La infraestructura es un conjunto de bienes de capital que sustenta la estructura productiva y contribuye a mejorar de forma efectiva las relaciones sociales, las actividades económicas individuales y colectivas, y los intercambios de bienes y servicios (Pópulo et al., 2002). Gestión de recursos. - el manejo de los recursos sean humanos, tecnológicos, financieros o de inventario es un 
punto crítico para que debe manejar las entidades financieras rurales para ser eficiente y eficaz basado en una planificación previa.

Equipos de comunicación. - son las acción conjuntas de todos los departamentos que permite al cliente sentirse identificado con los servicios ofrecido junto al valor agregado que los diferencia; con el objetivo primordial de brindar servicios de calidad; esto puede ser mediante publicidad en medios masivos, complementado con los medios sociales o los tradicionales. Facilidades de pago. - estrategias de políticas flexibles específicas para que los clientes se sientan a gusto y regresen a la empresa.

\section{Tecnología}

Análisis de las herramientas de monitoreo para evaluar la calidad del servicio que se oferta y brinda a los clientes o consumidores, tiene como base los sistemas informáticos en la página web, medios sociales y las apps propias de la empresa; y el soporte al seguimiento al cliente que maneja quejas, reclamos, sugerencias, inquietudes, consultas y manejo de comentarios.

Sistemas informáticos. - almacenaje y procesar información relevante incide en la toma de decisiones empresariales, orientados a potenciar la productividad. Este aspecto se puede aprovecharlos siguientes para las cooperativas rurales pertenecientes a la provincia de Tungurahua.

- Página web, brinda información precisa de la empresa, servicios y contactos. Su presentación tiene que ser atractiva visualmente y con estilo propio, incorporando lo colores corporativos, slogan y logotipo de la empresa.

- Apps o aplicaciones móviles, aportan asesoría directa y ágil en tiempo real al consumidor o cliente

- Medios sociales, irreemplazable forma de comunicación e interacción con el cliente, las más usadas son los blogs, redes sociales y App móviles (se divide en plataformas de pago y gratuitas).

- Sistemas de pago virtual o sistemas de pagos electrónicos, facilita los pagos por los servicios. Como son medios de pago offline como depósito, transferencia bancaria o pagos contra reembolsos y los medios de pago online como pagos con tarjeta de crédito, Pay Pal. Brindan seguridad también son confiables, permite el rastreo de los productos y opciones de devoluciones o reembolsos.

Seguimiento al cliente. - son las acciones claves no acosadoras de interacción con los clientes, que generaran fidelización, esta son:

- Soporte de quejas, reclamos y sugerencias, esto se puede realizar por llamadas telefónicas y emails, permite percibir su satisfacción con los servicios, promoción de descuentos u ofertas de otros servicios; de igual forma recordar fechas especiales para los clientes.

- Soporte de inquietudes y consultas, da respuestas a preguntas que el cliente posea comúnmente, con respecto a los servicios, precios e información básica de la 
empresa. En la actualidad se implanta los Chatbots, que son programas informáticos de soporte para interactuar con el cliente a través de conversaciones online.

- Manejo de comentarios negativos en medios sociales, es crucial para la reputación de la empresa. Se investigan los incidentes negativos, conversar con el personal para obtener más detalles, aclarar lo sucedido, no borrar los mensajes, responderlos públicamente y dar seguimiento al caso.

Herramientas de monitoreo. - tecnóloga que facilita el almacenaje y evaluación información, para cuantificar y cualificar datos para su análisis, ayuda a conocer la expectativa tanto del cliente como del empresario para las adaptaciones necesarias de su atención; para la evaluación continua se utilizan herramientas de monitoreo y control para medios sociales así como de medios publicitarios para su evaluación continua, se establece el comportamiento de los consumidores, sus necesidades, deseos, expectativas y perfil de consumo o adquisición de servicios; fijándose las acciones concretas para posicionar una empresa de servicios.

Indicadores. - con base a la información almacenada se elaboraran indicadores propios de satisfacción del cliente, seguimiento al cliente, como: expectativas, experiencia, percepción, quejas, reclamos y calidad en la atención estos a nivel interno. A nivel externo se maneja indicadores publicitarios y de marketing, como: visibilidad, conversión, tasa de permanencia, alcance de seguidores y fans y compromiso en medios digitales; todos encaminados a brindar un servicio de óptimo a los clientes.

\section{Sensaciones}

También se toma en cuenta las sensaciones, que involucran las características intrínsecas del servicio, las necesidades y expectativas en base a la confianza que poseen los clientes, y lo que generan percepciones positivas de acuerdo al valor percibido y de allí, la lealtad a la marca. Características intrínsecas del servicio. - son aspectos de intangibilidad (no se toca, pero se perciben los beneficios del servicio), inseparabilidad (no se pueden dividir), heterogeneidad (percepción y valoración diferente del servicio), ausencia de propiedad (nadie es dueño del servicio, no existía antes ni después).

Necesidades, expectativas y deseos. - al detectar estos tres factores las empresas pueden aprovechar esas oportunidades de negocio y ofrecer servicios que satisfagan esos vacíos. Dando a conocer a los clientes comportamientos e intenciones del proveedor de servicios, reflejan una visión positiva convirtiéndose en promotores y defensores.

Percepciones. - comprende dos grandes dimensiones, la intangibles (por ejemplo, atención del personal) y la tangibles (por ejemplo, instalaciones físicas). Definiendo así a la calidad del servicio como la percepción que tiene un cliente de la superioridad en el servicio que se recibe. Por ello, las empresas de servicio deben brindar una atención óptima al cliente, asesorarlo en todas sus dudas e inquietudes; preocupándose no solo de las instalaciones de la empresa sino también por sus empleados, por capacitarlos en áreas específicas de atención al cliente en forma personal y a través de medios digitales. 
Valor percibido. - es la comparación durante la transacción entre beneficios y costos, por lo que una misma persona podría evaluar el mismo servicio de forma diferente en distintas ocasiones.

Por lo cual, las cooperativas rurales deben cumplir con todo lo ofertado, ya que la satisfacción de los clientes se genera a partir de la satisfacción de sus expectativas.

Lealtad a la marca. - parte del proceso que involucra acciones de fidelización (felicitaciones por cumpleaños y fechas especiales, descuentos y nuevos servicios), esta fase comienza del desconocimiento total de la marca y de los productos que ofertan, después pasa por el reconocimiento del cliente o consumidor considera estos servicios nuevos en el momento de compra. Posteriormente, se da una preferencia por los consumidores y finalmente la lealtad, que se genera con vínculo estrecho con clientes. Se establecen estrategias concretas de lealtad que vinculen aspectos de comunicación para que los consumidores conozcan todos los servicios que una empresa oferta y sus beneficios.

\section{Exigencias}

Se establece los parámetros de identidad corporativa, relaciones internas, publicidad y comunicación integral; que conlleven a una percepción de la calidad del servicio y a un seguimiento posterior. De la forma en que se organicen y se y se dispongan para atender al cliente dependerá, en gran medida, que, en el momento de la verdad, el personal en contacto y los soportes físicos estén a la altura de las exigencias del cliente (Lara , 2002b). Identidad corporativa. - es la representación visual coordinado de signos visuales que son reconocidos instantemente, genera una opinión pública memorable. Es imprescindible la creación y manejo de un manual de identidad corporativa, donde establezcan los colores corporativos y dimensiones, usos correcto e incorrecto del logotipo en tarjetas de presentación, hojas, carpetas y sobres membretados, los uniformes y la publicidad. Siendo así una guía para toda la empresa, sus colaboradores y en específico de los departamentos de marketing, publicidad, ventas y recursos humanos.

Percepción frente a un servicio percibido. - son las expectativas o experiencias que el cliente recibe por el servicio o espera recibir por parte de la institución u organización. Determina si el producto o servicio dados brindaron una experiencia desagradable; si se cumple con lo ofrecido para que el cliente se sienta satisfecho siendo un referente para otros clientes potenciales.; las cooperativas rurales satisfacen por medio de un trato cordial y amabilidad, utiliza un léxico además de un lenguaje con un tono de voz adecuado expectativas de los clientes por parte del personal.

Seguimientos posteriores. - Se pueden realizar encuestas de satisfacción del servicio presenciales y online después que el cliente ha adquirido el servicio, esto permite medir el grado de satisfacción de las necesidades de los clientes con respecto a los productos o servicios al relacionarlos con las opiniones, sugerencias y quejas que realiza. También, se debe enfocar en el uso de contenido valioso en medios digitales, realizar descuentos y 
ofertas a los clientes frecuentes, así como felicitaciones en días festivos y cumpleaños, para que los clientes sientan un ambiente de confianza y compromiso.

\section{Discusión}

Para generar calidad en los servicios financieros de las cooperativas rurales de la provincia de Tungurahua se debe tener en cuenta la innovación tecnológica que facilita el obtener beneficios y mejoras en los servicios financieros. Por lo cual la tecnología actualizada beneficia al recurso humano, procesos, organización y responsabilidad social. La tecnología ayuda en la rapidez y eficiencia de los procesos generando así la calidad en el servicio (Larrán et al., 2007).

Para los clientes el obtener calidad significa satisfacer con sus expectativas, es decir es un conjunto de aspectos visibles y no visibles los cuales pueden generar un valor añadido y lograr la satisfacción del cliente. Los aspectos físicos generan comodidad, orden y limpieza al momento del servicio proporcionado, por lo cual nos da calidad en el servicio (Lara, 2002a). Para muchas empresas cumplir y hasta exceder las expectativas de los clientes se ha convertido en un reto porque entregar un servicio de calidad y lograr fidelizar a los clientes es muy importante debido a que la imagen de las empresas es dada por el tipo de servicio que ofrecen así mismo tener tecnología actualizada ayuda a mejorar los procesos de las empresas logrando así tener un correcto manejo del tiempo para satisfacer a los clientes y por ende mejorando la imagen que la empresa tiene por entregar sus servicios.

Proveer un servicio en el tiempo prometido es importante para lograr tener clientes satisfechos porque el tiempo es lo que menos les gusta perder, así el servicio de calidad y el tiempo que se entrega el servicio al cliente aparece como un elemento diferenciador en el mercado por esta razón la importancia de tener empleados capacitados en atención al cliente solo así se lograra ser empresas competitivas y retadoras (de Diago, 2013).

\section{Conclusiones}

- La situación actual del mercado de las cooperativas rurales de Tungurahua determina que las cooperativas rurales realizan esfuerzos continuos para brindar a sus clientes la satisfacción, sin embargo, estos esfuerzos que realizan no son los suficientes, tras las nuevas e innovadoras mejoras que el mercado brinda día a día a medida que los medios tecnológicos avanzan y se convierte en una relación empresa-cliente más estrecha y cercana.

- El modelo que se propone se atribuye a la innovación tecnológica permanente para el mejoramiento de la calidad de servicios en las cooperativas rurales de Tungurahua, este modelo permite un seguimiento a los servicios que se ofertan, hace hincapié en aspectos tecnológicos y de monitoreo, integrando las dimensiones de credibilidad, actitud y afinidad, relaciones, material, tecnología que dan paso a las sensaciones y exigencias. 


\section{Referencias bibliográficas}

Acevedo, A., \& Linares, C. (2009). La resolución de problemas en el mundo de la empresa. Estudio exploratorio sobre relativismo decisional, 2.

Altamirano \& Freire (2019). La innovación tecnológica y su impacto en el mejoramiento de la calidad de servicios en las empresas de servicios de Tungurahua.

Ampuño, F., Cañizares, G., \& Raymond, V. (2015). El cheque como medio de pago en la era de la tecnología de la información. Caso Ecuador. Periodo 2007-2013. Observatorio de la Economía Latinoamericana, 208.

Arzola, M., \& Mejías, A. (2007). Modelo conceptual para gestionar la innovación en las empresas del sector servicios. Revista Venezolana de Gerencia, 12(37), 80-90.

Botero, M. M., \& Peña, P. (2006). Calidad en el servicio: El cliente Incógnito. Suma Psicológica, 217-228.

Cardona, D., Balza, F., \& Henriquez , G. (2017). Innovación en el sector de los servicios: aproximacion conceptual y revisión de su aporte a la economía. Universidad de la costa, 57-98.

Carlos Omelas, C. E., Montelongo Cortés, Y., \& Nájera Gallardo, M. (2010). La Calidad del Servicio de un Centro de Información. Ciencia Tecnológica, 5-9.

Corporación Tecnova UEE. (2011). La importancia de la vigilancia tecnológica. REVISTA CES PSICOLOGÍA, 1-2.

Cuevas, H. (2016). La influencia de la innovación tecnológica en la competitividad de las PYMES Manufactureras. Universidad Autonoma de Aguas Calientes, 2-15.

de Diago, B. (2013). Mystery shopping: indicadores de estudio en bibliotecas. Revista General de Información y Documentación , 369-386.

Eduarte Ramírez, A. (1999). Calidad en el servicio al cliente. Revistas de Ciencias administrativas y financieras de la seguridad social, 41-44.

Fernández, A. (2019). Inteligencia artificial en los servicios financieros. Boletín Económico, 5-10.

Ferrer, J., \& Martínez, J. (2006). RESPONSABILIDAD SOCIAL Y MERCADEO DE SERVICIOS OFRECIDOS POR LAS EMPRESAS DEL SECTOR METALMECÁNICO. CICAG, 1-19.

Gil Lafuente, A., \& Luis Bassa , C. (2011). Innovación centrada en el cliente utilizando el modelo de inferencias en una estrategia CRM. Investigaciones Europeas de Dirección y Economía de la Empresa, 15-32. 
Gómez, C. (2010). La relación cliente-agencia: visión de los clientes. Pensamiento \& Gestión, 25-53.

Gonzales, S., \& Vélez, J. (2015). La gestión administrativa y su incidencia en la cartera vencida. $U L V R, 13-50$.

Gutiérrez Rodríguez , P., Vázquez Burguete, J. L., \& Cuesta Valiño, P. (2010). Valoración de los factores determinantes de la calidad del servicio público local: un análisis de la percepción de los ciudadanos y sus repercusiones sobre la satisfacción y credibilidad. INNOVAR JOURNAL, 139-156.

Instituto Nacional de Estadísticas y Censos [INEC]. (1 de Junio de 2012). CIIU 4.0. Obtenido de Clasificación Nacional de Actividades Económicas: http://aplicaciones2.ecuadorencifras.gob.ec/SIN/descargas/ciiu.pdf

Jiménez Zarco, A. I., Martínez Ruiz, M. P., \& González-Benito, Ó. (2008a). Implicaciones de la orientación proactiva hacia el mercado, la cooperación y el uso de las TIC en los procesos de innovación de productos y servicios. Business Review, 54-67.

Lara López, J. R. (2002a). La gestión de la calidad en los servicios Conciencia Tecnológico. Ciencia Tecnológica, 0-19.

Lara López, J. R. (2002b). La gestión de la calidad en los servicios. Ciencia Tecnológica, 0 .

Larrán Jorge, M., \& Muriel de Los Reyes, M. J. (2007). La banca por Internet como innovación tecnológica en el sector bancario. Investigaciones europeas de dirección de la empresa, 145-153.

Lockward Dargam, A. (2011). El rol de la confianza en las organizaciones a través de los distintos enfoques o pensamientos de la administración. Ciencia y sociedad, 12.

Marino, F., \& Moreno, L. (2014). Actividad comercial en el exterior de las empresas . Publication, 33-60.

Núñez , J., \& Montalvo, L. (2015). La política de ciencia, tecnología e innovación en Cuba y el papel de las Universidades. Rev. Cubana de Educación superior, 6595. Obtenido de http://scielo.sld.cu/scielo.php?script=sci_arttext\&pid=S025743142015000100003

Núñez, J. (2013). La política de ciencia, tecnología e innovación en la actualización del modelo económico cubano. Economía y desarrollo, 35-75.

Pérez, C. (6 de 6 de 2018). Estratégias de comunicación para la adopción de servicios de banca por internet en el sector financiero de la provincia de Tugurahua. Estratégias de comunicación para la adopción de servicios de banca por internet 
en el sector financiero de la provincia de Tugurahua. Ambato, Tungurahua, Ecuador: Repositorio UTA.

Pópulo, M., Romero, P., \& Delgado, G. (2002). La exposición universal de Sevilla 1992: Efectos sobre el crecimeinto económico Andaluz. España: Universidad de Sevila: Fundación Focus Abengoa.

Rodríguez, J. (2015). Estrategia empresarial e innovación tecnologicas de las industrias Brasileñas. Sistema de Información científica, 65-84. Obtenido de http://www.redalyc.org/html/877/87742317005/

Rodríguez, L., Góngora, W., Escalona, A., Cobos, D., Bastidas, S., Loustanau, C., \& Gonzales, A. (2015). Resultados del proceso productivo de la solución concentrada de propóleos, impacto de la innovación tecnológica. Ciencias Quimicas, 139-147. Obtenido de https://revistas.unal.edu.co/index.php/rccquifa/article/view/56288

Salazar, P. (2011). La importancia de la satisfacción del usuario. Documentación de las Ciencias de la Información, 349.

Solórzano, G., \& Aceves, J. (2013). Importancia de la calidad del servicio al cliente para el funcionamiento de las empresas. El buzón de Pacioli, 4-14.

Tamarit, J. (2015). La transformación de los servicios hacia la calidad de vida, una iniciativa de innovación social de FEAPS. Siglo Cero, 36-41. Obtenido de http://revistas.usal.es/ revistas_trabajo/index.php/02101696/article/view/scero20154634771

Tumino, M., \& Komiejczuk, V. (2012). Acción y Reflexión: Actitud del Estudiante hacia el Aprendizaje y el Servico. Enfoques, 53-76.

Zahera Pérez, M. (2003). La gestión de la innovación tecnológica (GIT) en la empresa. E-Deusto, 25, 16-20.

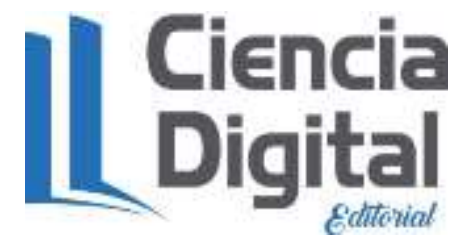




\section{PARA CITAR EL ARTÍCULO INDEXADO.}

Jiménez-Silva, W. R., Silva-Ordoñez, I. F., \& Padilla-Martínez, M. P. (2021). Calidad de los servicios financieros de las cooperativas rurales de la provincia de Tungurahua. AlfaPublicaciones, 3(4.1), 6-25. https://doi.org/10.33262/ap.v3i4.1.121

\section{Liencia}

El artículo que se publica es de exclusiva responsabilidad de los autores y no necesariamente reflejan el pensamiento de la Revista Alfa Publicaciones.

El artículo queda en propiedad de la revista y, por tanto, su publicación parcial y/o total en otro medio tiene que ser autorizado por el director de la Revista Alfa Publicaciones.
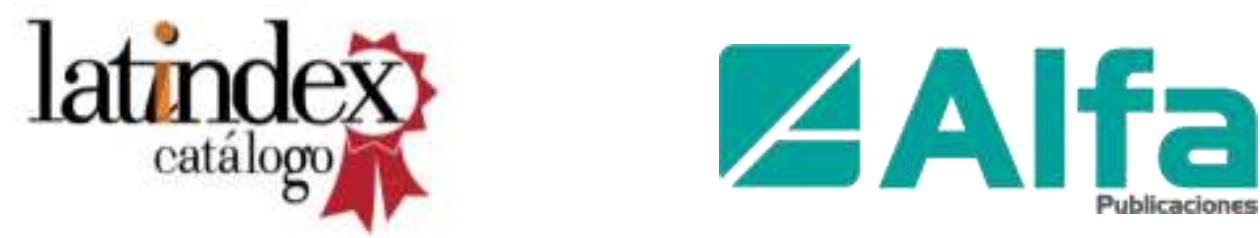